\title{
Mixed Response to Ipilimumab in a Melanoma Patient with Brain Metastases: Case Report and Review of the Literature
}

\author{
Georg Feldmann ${ }^{\mathrm{a}} \quad$ Peter Brossart ${ }^{\mathrm{a}} \quad$ Matthias Zipfel ${ }^{\mathrm{a}}$ \\ Marie von Lilienfeld-Toal ${ }^{a, b}$ \\ ${ }^{a}$ Department of Internal Medicine 3, Center of Integrated Oncology (CIO) Cologne-Bonn, \\ University Hospital of Bonn, Bonn, and ${ }^{\mathrm{b}}$ Hematology/Oncology, Jena University Hospital, \\ Jena, Germany
}

\section{Key Words}

Ipilimumab $\cdot$ Melanoma $\cdot$ Brain metastases $\cdot$ Survival

\begin{abstract}
Management of patients suffering from metastatic malignant melanoma and brain metastasis remains challenging in routine clinical practice. The inhibitory anti-CTLA-4 antibody ipilimumab has recently been approved as second-line therapeutic option for melanoma patients. Increasing evidence suggests distinct therapeutic activity on central nervous system metastases, although this continues to be actively debated. Here, we present the case of a patient suffering from metastatic melanoma, including symptomatic brain metastasis, who showed a partial response to ipilimumab in extracranial tumor manifestations, while the disease was progressing intracranially. Subsequently, intracranial disease progression could be managed by local irradiation. An overview of currently available literature on the efficacy of ipilimumab in melanoma patients with central nervous system metastases is provided.
\end{abstract}

\section{Introduction}

The overall prognosis of patients with advanced melanoma and brain metastases is generally poor, and therapeutic options are limited [1,2]. Surgery or irradiation may be applied in some cases for the local control of metastatic growth within the central nervous system (CNS). Temozolomide has the potential to cross the blood-brain barrier and by now 
constitutes one of the most promising systemic treatment approaches for this group of patients in the Western world, although with overall response rates of approximately 10$15 \%$, only a small minority actually benefits in a clinically meaningful manner $[3,4]$. The human inhibitory anti-CTLA-4 antibody ipilimumab has recently been shown to induce a significant, durable response and to prolong overall survival in metastatic melanoma patients $[5,6]$. The therapeutic efficacy of ipilimumab has been proven in various organ sites including liver, lungs, adrenal glands, skin, lymph nodes and bone [7], but data on the safety and therapeutic efficacy of ipilimumab in brain metastases of malignant melanoma are limited, since the presence of brain metastases represented an exclusion criterion in most clinical studies in the past [8].

\section{Case}

In December 2011, a 69-year-old male patient was admitted to our institution for the evaluation of further therapeutic options of metastatic malignant melanoma. Previously, in March 2009, the diagnosis of an ulcerated nodular solid malignant melanoma of the left cheek (thickness $30 \mathrm{~mm}$, Clark level 5) with infiltration of the left parotid gland had been made. The neoplastic cells carried wild-type B-RAF alleles, but an oncogenic mutation in exon 2 of the N-RAS gene was found. The past medical history was unremarkable except for hypertension, for which antihypertensive combination therapy was given. In March 2009, the patient underwent surgical excision and neck dissection, during which a total of 16 lymph nodes were removed, which histologically did not show any signs of tumor invasion at that time. Subsequently, however, in October 2009, a soft tissue metastasis developed at the base of the resection area, which was treated with local irradiation over a course of 2 months. In December 2010, another subcutaneous metastasis was found above the left base of the mandible, which was surgically resected. Histopathological analysis showed clear resection margins.

Adjuvant immunotherapy with 3 million units of interferon- $\alpha$ thrice weekly was initiated in January 2011 but terminated in May 2011, when increasing serum concentrations of the tumor marker S100 indicated disease progression under therapy. Moreover, by this time, the patient had developed numbness and weakness of the right arm and left leg. A CT scan revealed newly developed lung metastases and lymph node metastases in the right hilar region as well as below the carina, and an MRI scan showed 2 new brain metastases - one in the left frontal region and another one in the area of the head of the right caudate nucleus.

After a course of stereotactic irradiation $(7 \times 5$ Gy, i.e. a cumulative dose of $35 \mathrm{~Gy})$, another MRI scan performed in December 2011 showed regression of the left frontal brain metastasis and constant size of the right caudate nucleus lesion.

Systemic therapy with temozolomide was started in August 2011. A restaging CT scan was performed in November 2011 and showed progressive disease under therapy, with an increase in the size of 2 lung metastases and a newly diagnosed lymph node metastasis in the left axilla. At this point, temozolomide therapy was abandoned and the patient was referred to our institution for evaluation of further therapeutic options.

Upon presentation at our clinic, the neurological symptoms had resolved completely, and the patient reported no relevant symptoms except for an overall feeling of fatigue and lack of energy. At this point in time, the patient was on continuous prophylactic steroid therapy with low-dose decortin. In December 2011, second-line therapy with ipilimumab was initiated at a dose of $3 \mathrm{mg} / \mathrm{kg}$ intravenously every 3 weeks for a total of 4 courses. The therapy was well tolerated without any signs of toxicity or tangible adverse effects. In 
particular, the patient did not develop diarrhea or other signs of autoimmune disease and showed no neurological deficits. Routine follow-up laboratory parameters remained mostly normal except for an isolated increase in serum lactate dehydrogenase (LDH) concentrations of up to $348 \mathrm{U} / \mathrm{l}$ during the initial 2 cycles of ipilimumab, which spontaneously resolved upon cessation of ipilimumab administration (fig. 1).

A follow-up CT scan performed in March 2012 demonstrated stable disease with respect to extracranial manifestations, with marked size regression of the mediastinal metastases as well as the left axillar lymph node metastasis and constant size of a right-cervical lymph node $(1.3 \mathrm{~cm}$ in diameter). Of interest, at this point, the tumor marker S100 had dropped from $0.24 \mu \mathrm{g} / \mathrm{l}$ before initiation of ipilimumab administration in December 2011 to $0.16 \mu \mathrm{g} / \mathrm{l}$ in April 2012 after 4 cycles of ipilimumab (fig. 1). However, an MRI scan performed in March 2012 revealed size progression of the brain metastasis in the area of the head of the right caudate nucleus, a newly developed metastasis in the left frontal region as well as several new small metastatic lesions in the left parietal region.

Therefore, administration of ipilimumab had to be stopped, and another round of percutaneous irradiation of the neurocranium was initiated $(5 \times 2$ Gy per week up to a total dose of $40 \mathrm{~Gy}$, i.e. a cumulative dose of $75 \mathrm{~Gy}$ ).

A repeat MRI scan done in October 2012 revealed a decrease of the cerebral metastases by $25-50 \%$ on average, without occurrence of any new cerebral metastases. A CT scan of the abdomen, thorax and neck performed in November 2012 showed a mixed response, with regression of some distant organ metastases, while others had progressed or newly developed. By November 2012, the tumor marker S100 had again risen to $0.43 \mu \mathrm{g} / \mathrm{l}$.

The patient is currently in a rather good clinical condition and able to perform the tasks of routine everyday life.

\section{Discussion and Review of Currently Available Literature}

Patients suffering from metastatic melanoma with brain metastases carry an exceptionally poor overall prognosis, and therapeutic options are limited [1, 2]. Limited clinical data exist on the therapeutic efficacy of the CTLA-4 antibody ipilimumab, which was recently shown to significantly prolong survival in patients with advanced melanoma with brain metastases. Ipilimumab is believed to confer therapeutic efficacy via modulation of the antitumor immune response with a relative abrogation of regulatory $\mathrm{T}$ cell function and expansion of CD8+ cytotoxic T cells, thereby inducing overall enhanced T cell-mediated tumor cell lysis. The CNS is generally considered an immunologically privileged site where immune reactions are mostly suppressed, and proteins including immunoglobulins are largely excluded from the CNS, so that from the very beginning there was considerable skepticism as to the therapeutic efficacy of ipilimumab on metastatic lesions within the CNS [9]. On the other hand, it has recently been shown that in certain cases activated $T$ cells have the potential to cross the blood-brain barrier and readily infiltrate neoplastic tumor tissues within the brain, giving rise to the possibility that substances that act through stimulation of $\mathrm{T}$ cell responses such as ipilimumab might in fact have a therapeutic effect on brain metastases [10].

Two case reports from different authors suggest the clinical efficacy of ipilimumab in melanoma patients with brain metastases. Hodi et al. [11] for the first time reported the administration of ipilimumab in a 63-year-old female patient with neurologically symptomatic melanoma metastases to the CNS. This patient did not show an objective response to ipilimumab as defined by the RECIST criteria, but the pathological review of resected brain 
metastasis tissue revealed a predominantly cytotoxic antitumor immune response in the CNS with a predominance of infiltrating CD8+ lymphocytes and paucity of FoxP3+ regulatory cells following CTLA-4 blockade [11]. Schartz et al. [8] reported complete remission in a 75year-old melanoma patient with brain metastases upon treatment with ipilimumab, without occurrence of any major unexpected adverse effects other than recurrent eczema-like eruption. Of interest, complete remission was reached only 2 years after initiation of ipilimumab treatment in this patient, suggesting that sufficient time is required to adequately judge therapeutic efficacy.

Another group recently reported the case of a patient with malignant melanoma metastatic to the brain who survived for over 40 months under therapy with ipilimumab plus vemurafenib [12].

Of interest, Du Four et al. [13] reported focal radiation necrosis of the brain as a possible adverse effect in patients suffering from melanoma metastatic to the CNS who received ipilimumab plus local irradiation.

In 2012, Margolin et al. [14] reported on a prospective nonrandomized phase II clinical trial of ipilimumab for patients with advanced melanoma and brain metastases. In this study, 5 of $51(10 \%)$ neurologically asymptomatic patients who did not require supportive treatment with steroids showed a partial response, and another $4(8 \%)$ showed stable disease at 12 weeks after initiation of ipilimumab treatment given as intravenous injections of $10 \mathrm{mg} / \mathrm{kg}$ every 3 weeks for a total of 4 doses. Of note, this study also included 21 patients receiving systemic steroid treatment due to neurologically symptomatic brain metastases. Out of these, 1 patient (5\%) showed a partial response to ipilimumab treatment at week 12 , and the other 20 patients showed progressive disease under therapy [14, 15]. Commenting on this report, Nieder [16] rightly pointed out that potential effects on the response of brain metastases conferred by concomitant or previous CNS irradiation could not be ruled out in this study.

Di Giacomo et al. [17] recently reported that out of 20 melanoma patients with brain metastases treated with ipilimumab plus fotemustine combination therapy, 10 (50.0\%, 95\% CI 27.2-72.8) achieved immune-related disease control. Out of these, 5 had reduced or stabilized brain disease and 5 had brain metastases that became undetectable by scan.

Similarly, the case we have presented here confirms that treatment with ipilimumab should be considered as a suitable and safe second-line therapeutic option for patients with melanoma metastatic to the brain. In this case, the antibody was well tolerated and no ill effects were observed as a result of ipilimumab administration. Interestingly, a dramatic shrinkage of extracranial disease manifestations was observed after 12 weeks (i.e. 4 cycles) of ipilimumab therapy accompanied by a significant decrease in serum concentrations of LDH as well as of the tumor marker S100, indicating a decrease in the overall tumor burden. However, with respect to intracranial disease manifestations, the patient showed progressive disease at week 12 and thus required additional CNS irradiation in the subsequent course. Whether intracranial disease progression was due to the fact that in this case the intracranial tumor lesions represented a selection of more aggressive and more therapyresistant subclones or that the therapeutic effect was ameliorated due to an overall lack of efficacy in the CNS remains a matter of speculation at this point. More importantly, however, since this constellation formally indicated progressive disease, continuing the administration of ipilimumab was not possible, since this would have been outside the currently approved range of indications.

Nevertheless, overall, this patient clearly profited from the administration of ipilimumab-based therapy supported by CNS irradiation. He is currently alive and well almost 4 years after the initial diagnosis of malignant melanoma or 1.5 years after the first appear- 
ance of brain metastases. Based on the promising extracranial effects of the initial 4 cycles of ipilimumab therapy, the continuation of antibody therapy until disease progression is currently considered.

Taken together, the case presented here exemplifies several points that should be taken into account when treating melanoma patients with CNS metastases. Firstly, there is now little doubt that ipilimumab should be considered as a viable and safe potential therapeutic option for second-line treatment in this particular group of patients. Of note, this can also be possible in cases where brain metastases have already become symptomatic, as is the case in the example presented here. Secondly, even patients with limited or absent control of CNS metastatic growth might still significantly profit from the administration of ipilimumab, since a combination with local irradiation is usually possible and provides an additional powerful tool to interrupt intracranial disease progression. Thirdly, sufficient time is required in order to be able to correctly judge the therapeutic response in this unique subset of patients. The currently recommended minimum of 4 treatment cycles, which is mainly based on experience in melanoma patients without CNS metastases, might not be enough in the unique setting of brain metastases. In cases like the one presented here, we might thus, in the current situation, have to withdraw a therapeutic component, although the patient might potentially still profit from continued application. Thus, we feel that the generation of more prospective data is urgently required in the form of controlled clinical trials specifically addressing the question of optimal dosing and duration of ipilimumab administration in combination with local irradiation in melanoma patients with brain metastases.

\section{Acknowledgement}

G.F. was supported by the German Cancer Foundation (Deutsche Krebshilfe) grants No. 109215 and 109929 as well as by the European Community's Seventh Framework Program (FP7-2007-2013) under grant agreement HEALTH-F2-2011-256986.

\section{Disclosure Statement}

The authors state that there is no conflict of interest that might impair the impartiality of this scientific work.

\section{References}

1 Nikolaou VA, Stratigos AJ, Flaherty KT, Tsao H: Melanoma: new insights and new therapies. J Invest Dermatol 2012;132(3 Pt 2):854-863.

-2 Preusser M, Capper D, Ilhan-Mutlu A, Berghoff AS, Birner P, Bartsch R, Marosi C, Zielinski C, Mehta MP, Winkler F, Wick W, et al: Brain metastases: pathobiology and emerging targeted therapies. Acta Neuropathol 2012;123:205-222.

-3 Kirkwood JM, Bastholt L, Robert C, Sosman J, Larkin J, Hersey P, Middleton M, Cantarini M, Zazulina V, Kemsley K, Dummer R: Phase II, open-label, randomized trial of the MEK1/2 inhibitor selumetinib as monotherapy versus temozolomide in patients with advanced melanoma. Clin Cancer Res 2012;18:555567.

4 Patel PM, Suciu S, Mortier L, Kruit WH, Robert C, Schadendorf D, Trefzer U, Punt CJ, Dummer R, Davidson N, Becker J, et al: Extended schedule, escalated dose temozolomide versus dacarbazine in stage IV melanoma: final results of a randomised phase III study (EORTC 18032). Eur J Cancer 2011;47:1476-1483.

$>5$ Robert C, Thomas L, Bondarenko I, O'Day S, Weber J, Garbe C, Lebbe C, Baurain JF, Testori A, Grob JJ, Davidson N, et al: Ipilimumab plus dacarbazine for previously untreated metastatic melanoma. N Engl J Med 2011;364:2517-2526. 
-6 Hodi FS, O’Day SJ, McDermott DF, Weber RW, Sosman JA, Haanen JB, Gonzalez R, Robert C, Schadendorf D, Hassel JC, Akerley W, et al: Improved survival with ipilimumab in patients with metastatic melanoma. N Engl J Med 2010;363:711-723.

>7 Hodi FS, Butler M, Oble DA, Seiden MV, Haluska FG, Kruse A, Macrae S, Nelson M, Canning C, Lowy I, Korman $\mathrm{A}$, et al: Immunologic and clinical effects of antibody blockade of cytotoxic T lymphocyte-associated antigen 4 in previously vaccinated cancer patients. Proc Natl Acad Sci USA 2008;105:3005-3010.

-8 Schartz NE, Farges C, Madelaine I, Bruzzoni H, Calvo F, Hoos A, Lebbe C: Complete regression of a previously untreated melanoma brain metastasis with ipilimumab. Melanoma Res 2010;20:247-250.

$>9$ Carson MJ, Doose JM, Melchior B, Schmid CD, Ploix CC: CNS immune privilege: hiding in plain sight. Immunol Rev 2006;213:48-65.

10 Prins RM, Vo DD, Khan-Farooqi H, Yang MY, Soto H, Economou JS, Liau LM, Ribas A: NK and CD4 cells collaborate to protect against melanoma tumor formation in the brain. J Immunol 2006;177:8448-8455.

11 Hodi FS, Oble DA, Drappatz J, Velazquez EF, Ramaiya N, Ramakrishna N, Day AL, Kruse A, Mac Rae S, Hoos A, Mihm M: CTLA-4 blockade with ipilimumab induces significant clinical benefit in a female with melanoma metastases to the CNS. Nat Clin Pract Oncol 2008;5:557-561.

12 Balakan O, Suner A, Yigiter R, Balakan T, Sirikci A, Sevinc A: Long-term survival in metastatic malignant melanoma: ipilimumab followed by vemurafenib in a patient with brain metastasis. Intern Med 2012;51:2819-2823.

$\$ 13$ Du Four S, Wilgenhof S, Duerinck J, Michotte A, Van Binst A, De Ridder M, Neyns B: Radiation necrosis of the brain in melanoma patients successfully treated with ipilimumab, three case studies. Eur J Cancer 2012;48:3045-3051.

14 Margolin K, Ernstoff MS, Hamid O, Lawrence D, McDermott D, Puzanov I, Wolchok JD, Clark JI, Sznol M, Logan TF, Richards J, et al: Ipilimumab in patients with melanoma and brain metastases: an open-label, phase 2 trial. Lancet Oncol 2012;13:459-465.

15 Margolin K: Ipilimumab in a phase II trial of melanoma patients with brain metastases. Oncoimmunology 2012;1:1197-1199.

-16 Nieder C: Ipilimumab in patients with melanoma and brain metastases. Lancet Oncol 2012;13:e277, author reply e277-e278.

17 Di Giacomo AM, Ascierto PA, Pilla L, Santinami M, Ferrucci PF, Giannarelli D, Marasco A, Rivoltini L, Simeone E, Nicoletti SV, Fonsatti E, et al: Ipilimumab and fotemustine in patients with advanced melanoma (NIBITM1): an open-label, single-arm phase 2 trial. Lancet Oncol 2012;13:879-886. 


\section{Case Reports in Oncology}

\begin{tabular}{l|l}
\hline Case Rep Oncol 2013;6:229-235 & \multicolumn{2}{l}{} \\
\hline DOI: $10.1159 / 000351125$ & $\begin{array}{l}\text { C 2013 S. Karger AG, Basel } \\
\text { www.karger.com/cro }\end{array}$ \\
\hline
\end{tabular}

Feldmann et al.: Mixed Response to Ipilimumab in a Melanoma Patient with Brain

Metastases: Case Report and Review of the Literature
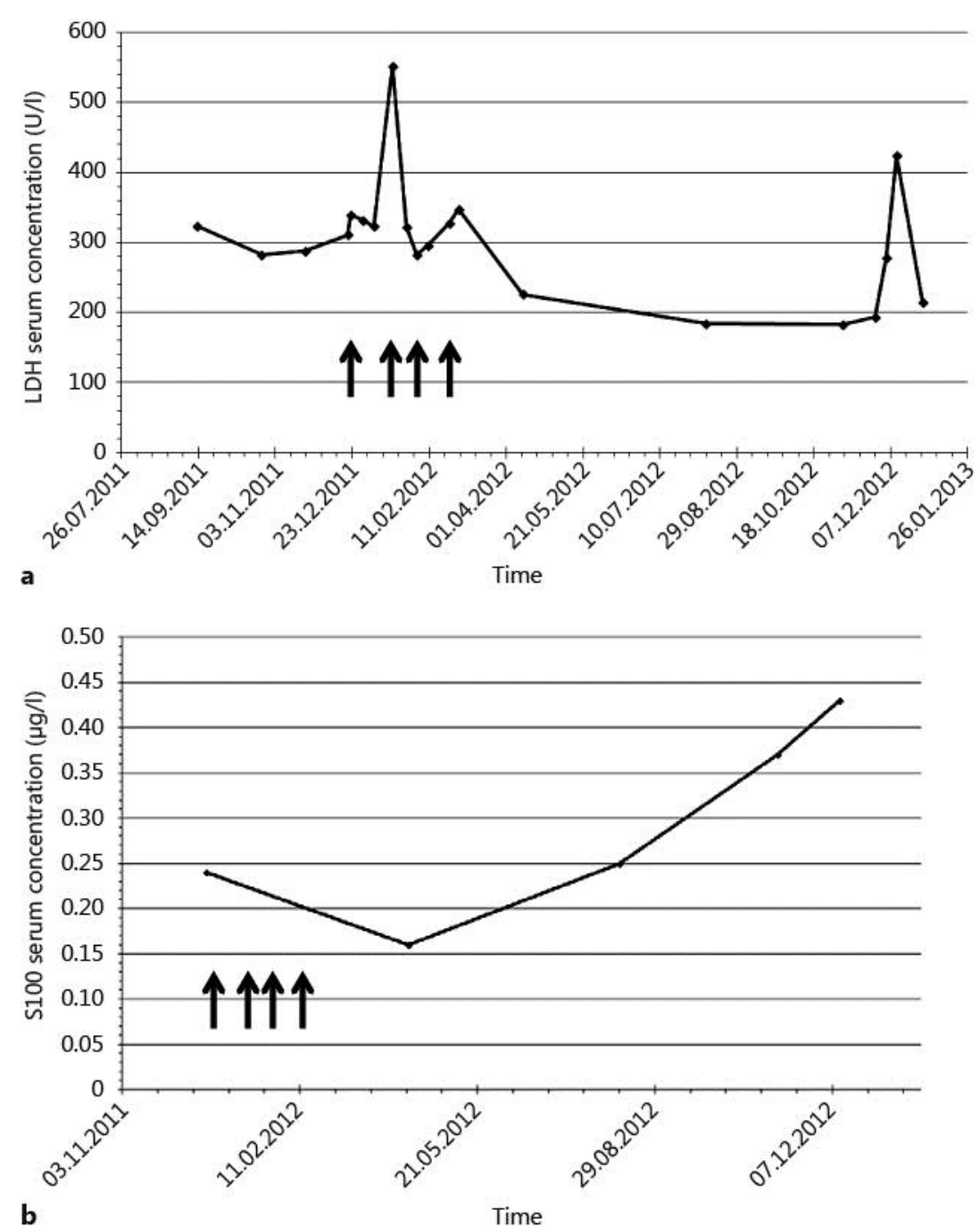

Fig. 1. a Administration of ipilimumab was accompanied by a temporary increase in serum LDH concentrations, followed by a slight but durable decrease over the next months. b Serum concentrations of the tumor marker S100 significantly dropped after administration of 4 cycles of ipilimumab, but subsequently started rising again after cessation of therapy, indicating a temporary initial reduction of total tumor burden. 